\title{
Establishing Random Aggregate Model of Recycled Concrete by MATLAB
}

\author{
Guangxu Lu, Lingqiang Yang* and Baoku Jiang \\ School of Civil Engineering and Architecture, University of Jinan 250022, China \\ ${ }^{*}$ Corresponding author
}

\begin{abstract}
Random aggregate model of recycled concrete is set up based on the understanding of recycled concrete mesostructure. First of all, the number of particles of all kinds of aggregate size was obtained on the $2 \mathrm{~d}$ and $3 \mathrm{~d}$ level by fuller grading theory and navarre gradation formula, then, Using carlo method ,the specific placement algorithm is developed based on the MATLAB language to generate the two-dimensional circular, three-dimensional ball geometric model of random aggregate. Finally, combining with the characteristics of recycled concrete, the simplified model of recycled concrete is put forward by improving the geometry model of random aggregate of ordinary concrete .The establishment of a typical two-dimensional circular, three-dimensional ball geometric model is completed in the ANSYS.
\end{abstract}

Keywords-recycled aggregate concrete; MATLAB; random aggregate model; mesostructure

\section{INTRODUCTION}

In recent years, the state has paid more and more attention to the sustainable use of natural resources, and the development of the construction industry should be transformed from the traditional extensive mode to the sustainable development mode. And recycled aggregate concrete can well adapt to the development model, abandoned concrete through a series of classification processing, can use again on the concrete block, also can be used as a kind of recycled aggregate instead of natural aggregate used in recycled concrete[1][2]. At present, many scholars have studied the recycled concrete and have achieved fruitful results. But the study of its performance are basically based on the basis of macroscopic test, generally do not consider its internal complex mesoscopic structure, the result is difficult to reveal the essence of the performance of recycled concrete. The difference of internal cause inevitably leads to the particularity of macroscopic appearance[3], so it is necessary to study the regenerative concrete in the detail level, and the numerical simulation method of ordinary concrete microstructure [4] provides the convenience for the study of the microscopic properties of recycled concrete.

At mesoscopic level, the arrangement and distribution of recycled aggregate in concrete structures have strong randomness, the content of the different and random geometric distribution, is a huge impact on the recycled concrete's performance. Therefore, it is very important to generate a microstructure model similar to the prototype regenerative concrete structure in the sense of probability statistics.

\section{BuILD A RANDOM CONCRETE MODEL OF RECYCLED CONCRETE}

\section{A. Theory of Aggregate Gradation}

Through the analysis of a large number of screening tests, Fuller[5] proposed the optimal matching curve of the maximum density. The equation is as follows.

$$
\mathrm{P}=100(\mathrm{D} / \mathrm{Dmax}) 1 / 2
$$

$\mathrm{P}$ means mass percent, Dmax means maximum aggregate size.

According to Walraven formula[6] the three-dimensional fuller aggregate gradation curve into two-dimensional plane aggregate diameter $\mathrm{D}<\mathrm{D}_{0}$ aggregate cumulative distribution probability problems. By (2), the number of aggregate particles of different particle sizes on the cross section can be calculated, and the number of particles can be calculated according to the percentage of aggregate.

$$
\begin{aligned}
& P_{c}\left(D<D_{o}\right)=P_{k}\left[1.065\left(\mathrm{D}_{0} / \mathrm{Dmax}\right)^{1 / 2}-0.053\left(\mathrm{D}_{0} / \mathrm{Dmax}\right)^{4}\right. \\
& \left.-0.0012\left(\mathrm{D}_{0} / \mathrm{Dmax}\right)^{6}-0.0045\left(\mathrm{D}_{0} / \mathrm{Dmax}\right)^{8}+0.0025\left(\mathrm{D}_{0} / \mathrm{Dmax}\right)^{10}\right]
\end{aligned}
$$

$\mathrm{P}_{\mathrm{k}}$ means aggregate volume accounts for the percentage of total concrete volume.

\section{B. Aggregate Random Drop Program Based on MATLAB}

There have been a lot of researches on the generation of the aggregate geometry model. Currently, the language that can be used to write the random drop program of aggregate is V-B, Fortran, MATLAB, etc. [7] Through the comparison, because of the

MATLAB can be called a lot of ready-made functions, can reduce the workload of programming, and can realize the visualization of computational results and programming, this paper select MATLAB and study the specific algorithm to realize the random placement of aggregate.

1) Assumption and principle of random aggregate placement: It is assumed that the aggregate is uniformly distributed[8] in the drop area, and the aggregate is reduced to a circle. The results show that the circular aggregate particles can cause errors due to differences in the shape of the actual particles, but the effect is negligible. And in the aggregate must meet the principle of incompatibility conditions between neighboring aggregate, and assume that each aggregate 
particles has certain influence scope, through calculation, the distance between each aggregate particles circle should satisfy (3)

$$
\mathrm{L}>\mathrm{L}_{0}=1.1\left(\mathrm{r}_{\mathrm{i}}+\mathrm{r}_{\mathrm{j}}\right) .
$$

2) Description of the aggregate random placement program [9]

a) 2-d aggregate random placement program.

b) Determine the boundaries, that is the drop area.

- Use MATLAB to write the program of randomly generating the center position of different radius.

- Determine the conditions for the first aggregate center to be satisfied:

$x(1)-r(1)>a \& x(1)+r(1)<b$

$y(1)-r(1)>c \& y(1)+r(1)<d$;

$z(1)-r(1)>e \& z(1)+r(1)<f$;

- Determine that each other aggregate center needs to meet the conditions:

$$
\begin{aligned}
& x(i)-r(i)>a \& x(i)+r(i)<b \\
& y(i)-r(i)>c \& y(i)+r(i)<d \\
& z(i)-r(i)>e \& z(i)+r(i)<f \\
& f o r j=1: i-1 \\
& \left((x(i)-x(j))^{2}+(y(i)-y(j))^{2}+(z(i)-z(j))^{2}\right)^{0.5}>1.1(r(i)+r(j))
\end{aligned}
$$

- Deterministic interrupt condition

- Produces a series of center coordinates.

- Call the function in MATLAB function library to make the aggregate graph.

\section{A random Aggregate Model of Recycled Concrete was Established}

1) Treatment of recycled aggregate: The main difference between recycled concrete and ordinary concrete is the difference of coarse aggregate, one is natural coarse aggregate and one is recycled coarse aggregate. Compared with natural aggregates, the recycled coarse aggregate has an interfacial transition zone, namely the old interface, and it is assumed that the outer layer of all recycled aggregate is coated with an old mortar, as shown in Figure 1, and the thickness is usually determined by the mortar content of recycled coarse aggregate.

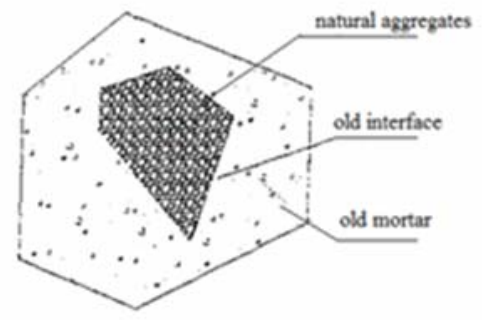

FIGURE I. RECYCLED COARSE AGGREGATE
According to the characteristics of recycled concrete, it is necessary to improve the random aggregate model of ordinary concrete. Think of the old mortar matrix as a ring shape. The thickness is $1.5 \mathrm{~mm}$ and the recycled coarse aggregate is reduced to two concentric circles[10]. The inner circle represents the natural aggregate, and the outer ring represents the old mortar, as shown in Figure 2

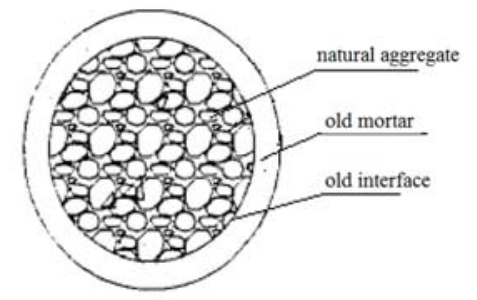

FIGURE II. RECYCLED COARSE AGGREGATE IN THE MODEL

2) Regenerated concrete interface treatment: In the simplified model, the recycled concrete has two interfaces: The interface between the recycled aggregates and old mortar is the old interface, the interface between the old mortar and the new hardened cement mortar is the new interface. At the microscopic level, because the thickness of the concrete interface layer is very small (20um 50um), it is extremely difficult and even impossible for the computer to conduct grid subdivision. To consider the amount of calculation, the interface layer thickness is bigger than the actual value (usually take $0.05 \sim 0.5 \mathrm{~mm}$ ), in general, the interface layer thickness is approximately uniform, therefore this article assumes that the interface layer thickness of different particle size of aggregate is the same, unity is $0.5 \mathrm{~mm}[11]$.

3) Build the ANSYS random aggregate model: The random aggregate model introduces the above points, after the improvement, the random aggregate model of recycled concrete was generated by MATLAB. Then it was imported into ANSYS, and the random aggregate model of recycled concrete containing old mortar thickness and interface thickness was obtained through Boolean operation.

\section{EXAMPLE}

In this paper, the two dimensional circular and threedimensional spherical random aggregate models of recycled concrete are taken as examples to verify the effectiveness of the proposed method.

\section{A. Calculation of Aggregate Particles}

1) Calculation of two dimensional aggregate particles: Take $100 \mathrm{~mm} \times 100 \mathrm{~mm} \times 100 \mathrm{~mm}$ cube specimen as an example. According to Fuller's aggregate grading curve, the gradation mode of recycled concrete was determined with medium aggregate $(D=20 \sim 30 \mathrm{~mm})$ and small aggregate $(\mathrm{D}=7 \sim 20 \mathrm{~mm})$. At the two dimensional level, the rectangular cross section of $100 \mathrm{~mm} \times 100 \mathrm{~mm}$ is taken to obtain the optimum structural strength and density. $\mathrm{P}_{\mathrm{k}}$ is calculated according to the percentage of coarse aggregate volume, which is 0.50 .The probability $\mathrm{Pc}$ of $\mathrm{D}<\mathrm{D}_{0}$ is calculated by using the 
Walraven formula, as shown in table 1. Then, (4) is used to solve the particle size of each particle size.

$$
\mathrm{N}_{\mathrm{i}}=\mathrm{S}_{\mathrm{i}} /\left(\pi \mathrm{D}_{\mathrm{i}}^{2} / 4\right) \quad \mathrm{S}_{\mathrm{i}}=\left(\mathrm{P}_{\mathrm{c}}{ }^{\mathrm{i}+1}-\mathrm{P}_{\mathrm{c}}{ }^{\mathrm{i}}\right) \mathrm{S}
$$

It was calculated that there were 10 small aggregates with a diameter of $10 \mathrm{~mm}$, and 5 small aggregates with a diameter of $16 \mathrm{~mm}$ and two medium aggregates with a diameter of $24 \mathrm{~mm}$ in the two-dimensional model.

TABLE I. THE VALUE OF THE PROBABILITY PC $(D<D 0)$

\begin{tabular}{|c|c|c|c|c|}
\hline $\mathbf{D}_{\mathbf{0}}(\mathbf{m m})$ & 30 & 20 & 12 & 7 \\
\hline $\mathrm{D}_{0} / \mathrm{Dmax}$ & 1 & 0.670 & 0.4 & 0.233 \\
\hline $\mathrm{P}_{\mathrm{c}} / \mathrm{P}_{\mathrm{k}}$ & 1 & 0.870 & 0.673 & 0.514 \\
\hline $\mathrm{P}_{\mathrm{c}}$ & 0.5 & 0.435 & 0.337 & 0.257 \\
\hline
\end{tabular}

2) Calculation of three - dimensional aggregate particles: Take $150 \mathrm{~mm} * 150 \mathrm{~mm} * 150 \mathrm{~mm}$ cube specimen as an example. According to Fuller's aggregate grading curve, the gradation mode of recycled concrete was determined with medium aggregate $(D=20 \sim 30 \mathrm{~mm})$ and small aggregate $(D=10 \sim 20 \mathrm{~mm})$. From (1), the occurrence probability $\mathrm{P}$ of $\mathrm{D}<\mathrm{D}_{0}$ is obtained, as shown in table 2.

TABLE II. THE VALUE OF THE PROBABILITY P (D<D0)

\begin{tabular}{|c|c|c|c|}
\hline $\mathbf{D}_{\mathbf{0}}(\mathbf{m m})$ & 30 & 20 & 10 \\
\hline P(Mass percent) & 100 & 81.6 & 57.7 \\
\hline
\end{tabular}

In the medium aggregate $(D=20 \sim 30 \mathrm{~mm})$, the average particle diameter $\mathrm{D} 1=24 \mathrm{~mm}$, and the average particle diameter of $\mathrm{D} 2=15 \mathrm{~mm}$ in the small aggregate $(\mathrm{D}=10 \sim 20 \mathrm{~mm})$. Concluded from table 2 , size of $20-30 \mathrm{~mm}$ aggregate quality percentage is $18.4 \%$, size $7-20 \mathrm{~mm}$ aggregate quality percentage is $23.9 \%$, Pk as coarse aggregate volume accounted for $43 \%$ of total volume calculation, according to the equation 4 to solve each size number of particles.

It was calculated that there were 188 small aggregates with a diameter of $15 \mathrm{~mm}$, and 35 medium aggregates with a diameter of $24 \mathrm{~mm}$ in the three-dimensional model.

\section{B. Drawing of Random Aggregate Figures}

1) Two-dimensional random aggregate graph: Using MATLAB programming to draw the $2 \mathrm{~d}$ aggregate graph automatically, its program source code is:

$\%$ function $[\mathrm{S}]=\mathrm{RC}(\mathrm{Num}, \mathrm{xmax}, \mathrm{ymax})$

$\mathrm{rl}=\left[\begin{array}{lll}0.012 & 0.008 & 0.005\end{array}\right] ; \% \%$ optional radius $\mathrm{r}$

num $=\left[\begin{array}{lll}2 & 5 & 10\end{array}\right] ; \% \%$ set the number of circles with an initial radius of $r$

numc $=\left[\begin{array}{lll}0 & 0 & 0\end{array}\right] ; \% \%$ The number of circles with an initial radius of $r$

rnum=[1 2 3 3$] ; \% \%$ The radius of the subscript

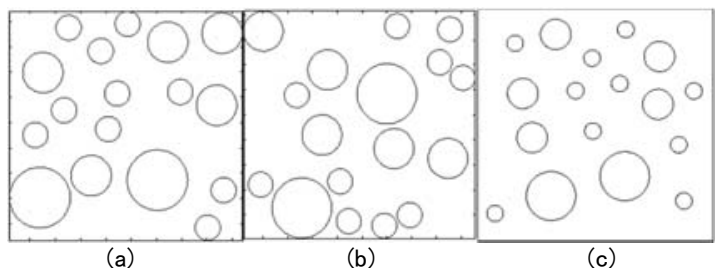

FIGURE III. TWO-DIMENSIONAL RANDOM AGGREGATE GEOMETRIC DISTRIBUTION IN MATLAB

2) Three-dimensional random aggregate graph: Program source code is:

\%function $[\mathrm{S}]=\mathrm{RC}(\mathrm{Num}, \mathrm{xmax}, \mathrm{ymax}, \mathrm{zmax})$

$\mathrm{rl}=\left[\begin{array}{ll}0.012 & 0.0075\end{array}\right] ; \% \%$ optional radius $\mathrm{r}$

num $=[35188] ; \% \%$ set the number of circles with an initial radius of $r$

numc $=\left[\begin{array}{ll}0 & 0\end{array}\right] ; \% \%$ The number of circles with an initial radius of $r$

rnum $=\left[\begin{array}{ll}1 & 2\end{array}\right] ; \% \%$ The radius of the subscript

It can be seen from the figure, under the same aggregate percentage between aggregate distribution model has a certain randomness, reflect the actual properties of gravel aggregate random distribution, which is one of the leading causes of concrete strength in discrete. The program is simple to run, which can show the randomness and feasibility of the actual aggregate distribution. Figure 3-c and 4-c are selected to generate the random aggregate model of recycled concrete.

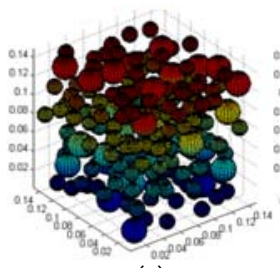

(a)

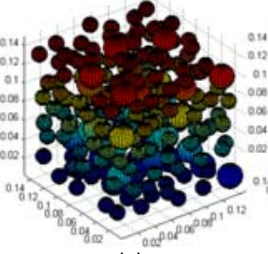

(b)

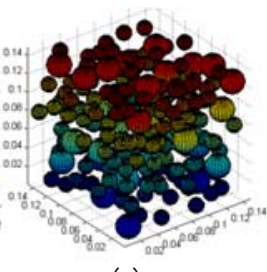

(c)
FIGURE IV. THREE-DIMENSIONAL RANDOM AGGREGATE GEOMETRIC DISTRIBUTION IN MATLAB

3) The generalization of algorithm application: By figure 5 , the application of MATLAB programming, besides can produce concrete cubic block model, the drawing can also be used for concrete specimen model, and has good feasibility, meet the random aggregate.

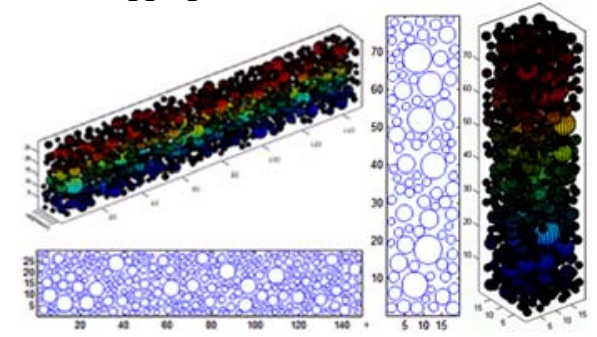

FIGURE V. CONCRETE BEAM MODEL AND CONCRETE COLUMN MODEL 


\section{Build the ANSYS Random Aggregate Model}

1) Random aggregate model of two - dimensional recycled concrete: A typical random aggregate model of $2 \mathrm{~d}$ recycled concrete is generated in ANSYS, as shown in Figure 6. It can be seen from the figure that the random aggregate model is composed of aggregate, old cement mortar, new cement mortar, the interface between the mortar matrix and the new hardening of cement mortar and the interface between recycled aggregate and the old cement mortar matrix.
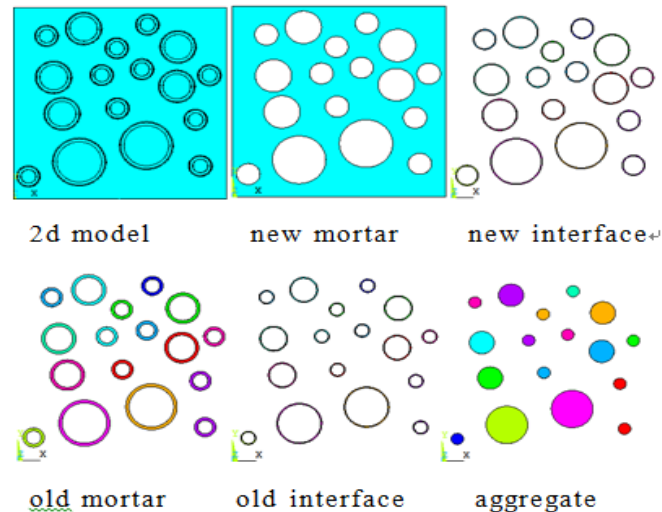

FIGURE VI. 2D RANDOM AGGREGATE MODEL

2) Random aggregate model of three-dimensional recycled concrete: Generated by ANSYS three-dimensional recycled concrete random aggregate model, as shown in figure 8 , can see clearly from the table, the three-dimensional model of random aggregate on the number and complexity are much bigger than the two-dimensional random aggregate model. In order to be intuitive to see the model of five units: aggregate, the old cement mortar, the new cement mortar, the interface between the mortar matrix and the new hardening of cement mortar and the interface between recycled aggregate and the old cement mortar matrix, five times to generate the model diagram of each unit, as shown in figure .
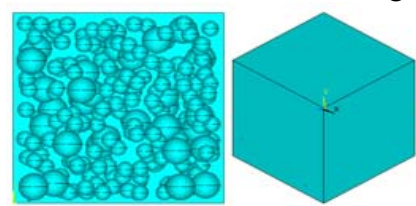

$3 \mathrm{~d}$ model new mortar

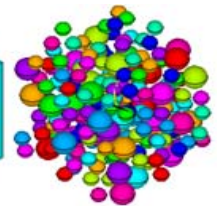

new interface

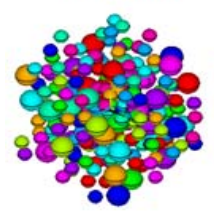

old morta

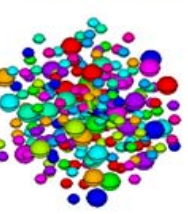

old interface

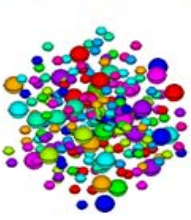

aggregate
FIGURE VII. 3D RANDOM AGGREGATE MODEL

\section{CONCLUSION}

Through MATLAB aggregate random delivery program, we obtain the aggregate random delivery of $2 \mathrm{~d}$ and $3 \mathrm{~d}$ model and has good feasibility, which laid a foundation for the next step of mesoscopic mechanics numerical analysis, but there are still some deficiencies:

- The aggregate lacks the randomness in shape, and is only simulated with a circle or sphere, which is different from the actual aggregate shape.

- The random delivery program increased the difficulty of the high content of aggregate model, because in the process of random delivery, first put in the position of aggregate has been identified, the subsequent aggregate cannot like the actual concrete pouring through vibration into the space between the aggregate.

\section{ACKNOWLEDGMENT}

This work was financially supported by the Shandong Natural Science Foundation (ZR2014EL038). Ling-qiang Yang is responding author.

\section{REFERENCES}

[1] Xiao Jianzhuang. Recycled concrete. [M].China Architecture \& Building Press, 2008.

[2] Oikonomou N.D. Recycled concrete aggregates. Cement\&ConcreteComposites,2005,27(2):315-31.

[3] Tang Chunan, Zhu Wancheng. Concrete damage and fracture numerical test.[M].Beijing: Science press,2003.

[4] Xia Xiaozhou. Study on the numerical simulation and macroscopic mechanics of concrete.[D].Nanjing:Hohai university doctoral dissertation,2007.

[5] Peng Yijiang, Li Baokun, Liu Bin.Numerical simulation of meso-level mechanical properties of roller compacted concrete[J].Journal of Hydraulic Engineering,2001,6(10):19-22.

[6] J.C. Walraven, H.W. Reinhard. Theory and Experiments on the Mechanics Behavior of Cracks in Plane and Reinforced Concrete Subject to Shear loading[J].HERON,1991,26(IA):26-33.

[7] XU Bo. Numerical Simulation and Performance Analysis of Concrete Based on Material Mesostures[D].Zhejiang University,2008.

[8] QH Gao , ZQ Guan, YX Gu, YH Yin.Automatic generation of finite element model for concrete aggregate [J]. Journal of Dalian University of Technology, 2006, 46(5).

[9] Gao L J, Liu X J, Wang Y M. Numerical Simulation for Random Embarking of Aggregate with Two-dimensional Meso-structure of Concrete Based on Matlab[J]. Journal of Hunan Institute of Engineering, 2011.

[10] Du Jiangtao. Stress - strain relationship test and numerical simulation of regenerated concrete uniaxial stress.[D].Tongji university master's thesis.,2008.

[11] Huai fa M A, Chen H Q, Bao kun L I. Review on micro-mechanics studies of concrete[J]. Journal of China Institute of Water, 2004. 